\title{
Nuclear transport factor directs localization of protein synthesis during mitosis
}

\author{
Geert van den Bogaart, Anne C. Meinema, Victor Krasnikov, Liesbeth M. Veenhoff and Bert Poolman
}

Nat. Cell Biol. 11, 350-356 (2009); published online 8th February 2009; corrected online 12th March 2013

In a follow-up study of our paper, we discovered that the Kap104-GFP strain obtained from Invitrogen and used in the published study is a mixture of three different strains, namely Fir1-GFP, Doa4-GFP and Kap104-GFP. The initial PCR check, using extracted chromosomal DNA and genespecific primers, identified the gene encoding Kap104-GFP, and immunoblot analysis using GFP antibodies did not reveal the contamination because all three of these GFP-tagged proteins have a similar molecular mass, $126 \mathrm{kDa}, 132 \mathrm{kDa}$ and $131 \mathrm{kDa}$, respectively. However, upon prolonged cultivation, the Fir1-GFP strain outgrows the others, and as a result, we have mistakenly worked with this strain. Therefore, the fluorescence pattern was mistakenly interpreted as that of Kap104-GFP; Fig. 1 thus shows the localization of Fir1-GFP rather than Kap104-GFP, and these data are no longer relevant to the paper. The GFP signals in Figs 2a and $3 g$ are from Fir1 and not Kap104. The first lane in Supplementary Fig. S1f corresponds to Fir1-GFP rather than Kap104-GFP. The localization of Kap104 as presented in the model in Fig. 5 is no longer supported by the data.

The experiments presented in the published paper that do not use the contaminated Kap104-GFP strain are correct and have been reproduced since the publication of the original study. The following conclusions of the paper continue to stand: (1) Kap104 has a role in increased translation in the daughter cell; (2) Kap104 cargo, Nab2 and Nab4 are asymmetrically distributed in mother and daughter cells; (3) Nab2-derived reporter proteins (rgNLS-GFP) undergo cell-cycle dependent Kap104-mediated nuclear import; (4) translation sites predominate in the daughter cell. However, our model suggesting that asymmetric localization of Kap104 underlies the asymmetry in cargo distribution and translation is no longer supported by the data. Thus, we do not currently have a mechanistic explanation for the role of Kap104 in increased translation in the daughter cell. 